\title{
Neural basis of functional ultrasound signals
}

\author{
Anwar O. Nunez-Elizalde ${ }^{1 *}$, Michael Krumin ${ }^{1}$, Charu Bai Reddy ${ }^{1}$, Gabriel Montaldo ${ }^{2,3,4,5}$, \\ Alan Urban ${ }^{2,3,4,5}$, Kenneth D. Harris ${ }^{6}$, and Matteo Carandini ${ }^{1}$ \\ ${ }^{1} \mathrm{UCL}$ Institute of Ophthalmology, University College London, London, United Kingdom \\ ${ }^{2}$ Neuro-Electronics Research Flanders, Leuven, Belgium \\ ${ }^{3}$ Vlaams Instituut voor Biotechnologie (VIB), Leuven, Belgium \\ ${ }^{4}$ imec, Leuven, Belgium \\ ${ }^{5}$ Department of Neuroscience, KU Leuven, Leuven, Belgium \\ ${ }^{6}$ UCL Queen Square Institute of Neurology, University College London, London, United Kingdom \\ *Correspondence: anwar.nunez@ucl.ac.uk
}

\section{Summary}

Functional ultrasound imaging (fUSI) is a popular method for studying brain function, but it remains unclear to what degree its signals reflect neural activity on a trial-by-trial basis. Here, we answer this question with simultaneous fUSI and neural recordings with Neuropixels probes in awake mice. fUSI signals strongly correlated with the slow $(<0.3 \mathrm{~Hz})$ fluctuations in firing rate measured in the same location and were closely predicted by convolving the firing rate with a $2.9 \mathrm{~s}$ wide linear filter. This filter matched the hemodynamic response function of awake mouse and was invariant across mice, stimulus conditions, and brain regions. fUSI signals matched neural firing also spatially: recordings with two probes revealed that firing rates were as highly correlated across hemispheres as fUSI signals. We conclude that fUSI signals bear a simple linear relationship to neuronal firing and accurately reflect neural activity both in time and in space.

\section{Introduction}

Functional ultrasound imaging (fUSI) is an increasingly popular method for studying brain function (Macé et al., 2011a; Rabut et al., 2020). fUSI is appealing because it can measure changes in cerebral blood volume with high resolution, revealing how the activity of brain regions is affected by sensory stimuli (Aydin et al., 2020; Bimbard et al., 2018; Blaize et al., 2020; Boido et al., 2019; Gesnik et al., 2017; Koekkoek et al., 2018; Macé et al., 2011a, 2018; Provansal et al., 2021; Urban et al., 2015), and by brain state and behavior (Brunner et al., 2020; Dizeux et al., 2019; Ferrier et al., 2020; Osmanski et al., 2014; Rahal et al., 2020; Sans-Dublanc et al., 2020; Sieu et al., 2015). fUSI resolves spatial features in the order of $\sim 100 \mu \mathrm{m}$, up to a depth of $\sim 2 \mathrm{~cm}$ (Macé et al., 2011b). Thanks to these capabilities, fUSI can be applied to rodent brains to provide images of activity akin to those obtained in humans with functional magnetic resonance imaging (fMRI). Thus, in mice, fUSI seems ideally poised to bridge the divide between single neuron and whole-brain measurements.
However, the relationship between fUSI signals and neural activity is indirect, involving multiple intermediate steps: neurovascular coupling, ultrasound sensing, and signal processing. First, neuronal firing is linked to changes in blood oxygenation, flow, and volume through the complex process of neurovascular coupling (Attwell and ladecola, 2002; Drew, 2019; Hamel, 2006; Hillman, 2014; ladecola and Nedergaard, 2007; Nair, 2005; Pisauro et al., 2013; Turner et al., 2020; Winder et al., 2017). Second, these vascular changes involve movement of blood, causing a frequency shift in ultrasound echoes that can be measured through power Doppler ultrasound sensing (Rubin et al., 1994, 1995). Third, the power Doppler signals are distinguished from multiple, large sources of noise -- such as tissue movement -through numerous signal processing steps: typically, temporal high-pass filtering and spatiotemporal clutter filtering by removing the principal components with the strongest amplitude (Baranger et al., 2018; Demené et al., 2015; Macé et al., 2011a, 2013). 
Because of these intermediate steps, it is unclear to what degree, and at what temporal and spatial scales, fUSI signals truly measure neural activity on a trial-by-trial basis. At first sight, fUSI signals appear noisy, with large fluctuations over short time scales (e.g. $>10 \%$ over a few seconds) that vary across trials (e.g. Brunner et al., 2020). It is not clear to what extent this variability is due to the process of measurement and analysis of fUSI signals, and how much it is neural. Indeed, neural activity exhibits endogenous, ongoing fluctuations that are strongly correlated across neurons (Schölvinck et al., 2015), are associated with changes in brain state and body movement (Drew et al., 2019; Musall et al., 2019; Stringer et al., 2019), and are highly correlated across hemispheres (Drew, 2019; Fox et al., 2006, 2007; Mohajerani et al., 2010; Shimaoka et al., 2019). Perhaps the apparently noisy fUSI signals reflect these structured fluctuations in neural activity. Indeed, fUSI signals approximately resemble simultaneously recorded local field potentials (Bergel et al., 2018; Provansal et al., 2021; Sieu et al., 2015), which in turn reflect local neuronal firing (Buzsáki et al., 2012; Katzner et al., 2009).

Moreover, it is not clear whether the neural component of fUSI signals reflect neuronal spiking through a simple linear relationship and if this relationship differs across brains and brain regions. To a first approximation, neurovascular coupling is a linear process: hemodynamic signals can be predicted from neuronal firing by convolving firing rates with a hemodynamic response function (Boynton et al., 1996; Devor et al., 2005; Drew, 2019; Heeger and Ress, 2002; Logothetis et al., 2001; Martindale et al., 2003; Pisauro et al., 2013). The next step might also be linear: fUSI signals can be predicted from separately-measured hemodynamic signals (red blood cell velocity) through a linear transfer function (Aydin et al., 2020; Boido et al., 2019). Because a series of linear operations is itself linear, it is thus possible that the relationship between fUSI signals and neuronal firing is the result of simple convolution with a linear filter and that this relationship is fixed across brain regions and types of activity.
Here we answer these questions with simultaneous measurements of spikes and fUSI signals in awake mice. We performed these experiments in the awake brain to avoid the detrimental effects of anesthesia on neurovascular coupling (Pisauro et al., 2013). The results indicate that fUSI signals are closely related to neuronal firing, and the relationship between the two is well summarized by convolution with a hemodynamic response function. The transfer function introduces a lowpass filter, such that the relationship between fUSI signals and neuronal firing becomes progressively more accurate at slower time scales. Neural activity explains why fUSI signals correlate strongly across space and even across hemispheres: these correlations reflect true shared fluctuations in neural activity across brain locations and hemispheres.

\section{Results}

To measure neuronal firing during fUSI, we recorded from Neuropixels probes during sensory and spontaneous activity (Figure 1a-b). For each mouse, we determined the location of primary visual cortex (V1) by aligning fUSI images to the Allen Institute Brain Atlas (Wang et al., 2020) using a vascular atlas as an intermediate reference (Todorov et al., 2020). In each session, we inserted a Neuropixels probe (Jun et al., 2017) in a parasagittal trajectory and acquired a fUSI image coronally (Figure 1a). Mice viewed a gray screen (to measure spontaneous activity) or flashing checkerboards (to measure visual responses). This sequence was repeated after moving the fUSI transducer to an adjacent coronal slice (3-5 slices per session, typically $0.4 \mathrm{~mm}$ apart). At the end of a session we determined the location of the probe in the fUSI images by slowly extracting it while detecting its movement with fUSI (Figure 1b). 



b Voxels traversed by NPx

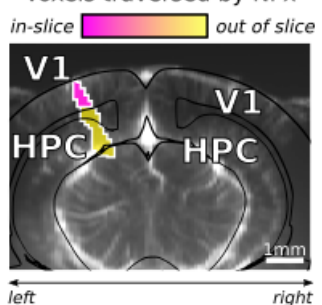

C Spikes


d
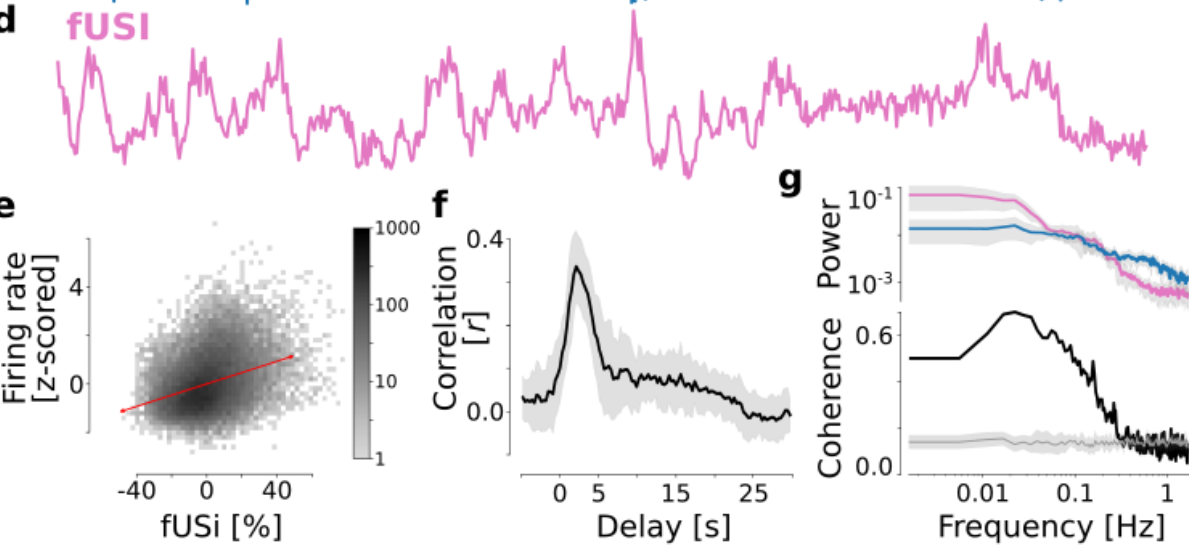

h $\quad$ Firing rate $\circledast \Lambda=$ Convolved firing rate
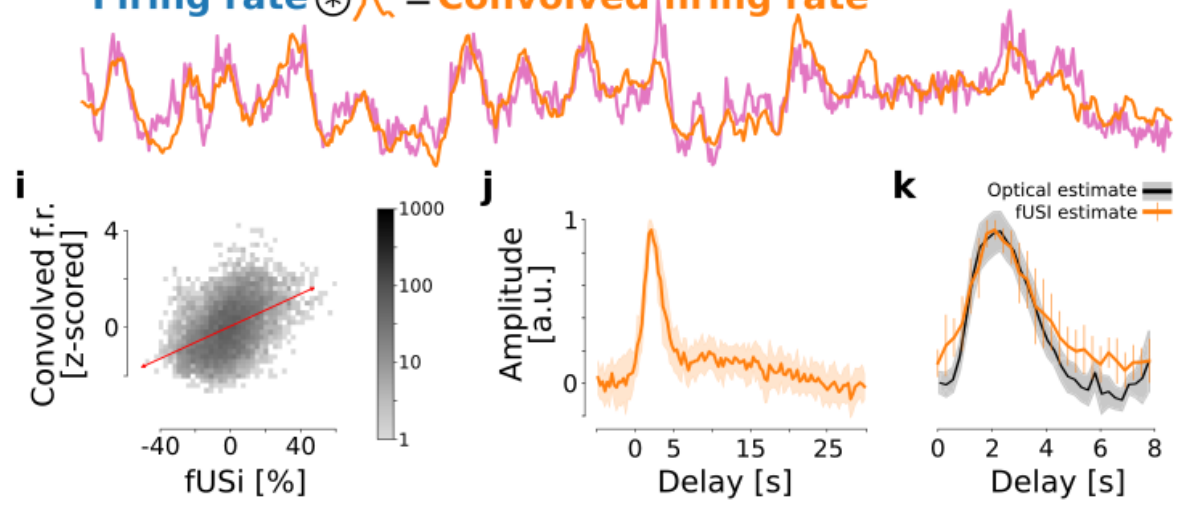

Figure 1. fUSI signal reflects temporally filtered firing rate during spontaneous activity. a. Schematic of simultaneous fUSI and electrophysiological recordings, showing primary visual cortex (V1) and hippocampus (HPC). b. A coronal fUSI slice with the reconstructed location of the Neuropixels probe. $c$. Spikes recorded in primary visual cortex (V1) in an example recording, showing spikes as a function of time and recording depth (top) and the resulting overall firing rate (bottom). d. fUSI signal measured simultaneously in the same location (average over 51 voxels). e. Comparison of fUSI signals and firing rate measured $2.1 \mathrm{~s}$ earlier (34 recordings in 5 mice), with best fitting line indicating correlation (red). f. Temporal cross-correlation between firing rate and fUSI signal, averaged across 34 recordings in 5 mice. Shaded region shows \pm 1 m.a.d. g. Power spectra (top) and spectral coherence (bottom) of firing rate and fUSI, averaged across recordings. $\boldsymbol{h}$. Convolving the firing rate with an optimal linear filter (orange) closely tracks the fUSI measurements (pink). i. Comparison of fUSI signals and convolved firing rate. $j$. Median linear filter across recordings ( \pm m.a.d. of 34 recordings in 5 mice). $\boldsymbol{k}$. The linear filter is similar to the optical estimate of the hemodynamic response function measured in awake mice (Pisauro et al., 2013).
The fUSI signals from visual cortex during spontaneous activity resembled the firing rate measured in the same location $\sim 2 \mathrm{~s}$ earlier, especially in its slow fluctuations (Figure 1c-f). After spike sorting, we computed the mean firing rate in all neurons (both single- and multi-unit clusters) recorded at the sites that intersected the fUSI slice (Figure 1c). We then compared this firing rate to the fUSI signal measured in the corresponding voxels (Figure 1d). The two traces appeared broadly similar and were significantly correlated (median $\rho$ $=0.34 \pm 0.08$ median absolute deviation, m.a.d., 34 recordings in 5 mice, Figure 1e). The crosscorrelation between firing rate and fUSI signals peaked at a delay of $2.10 \pm 0.3 \mathrm{~s}$ and had a full-width at half-height of $3.6 \pm 0.6 \mathrm{~s}$ ( \pm m.a.d., 34 recordings in 5 mice, Figure 1f). Thus, fUSI signals closely resemble the slow fluctuations in firing rate. 
Firing rate and fUSI signals were strongly correlated at low frequencies, and significantly correlated up to $\sim 0.3 \mathrm{~Hz}$ (Figure 1g). To estimate the correlation between fUSI and firing rate as a function of frequency, we computed their spectral coherence, i.e. their correlation as a function of frequency. Coherence was highest between 0.01 and $0.1 \mathrm{~Hz}$, with a median value of $0.59 \pm 0.03$ (m.a.d., 34 recordings in 5 mice), and gradually fell to chance levels (coherence of $0.14 \pm 0.03$ ) at a frequency of $0.32 \mathrm{~Hz}$. These results indicate that low frequency fluctuations in fUSI are mostly neural in origin, whereas fluctuations at higher frequencies are unrelated to neural activity and might thus best be discarded.

The precise relationship between fUSI signals and firing rate was well described by convolution with a linear filter (Figure $\mathbf{1 h}$-i). The cross-correlation between two signals reflects not only their interaction but also their individual autocorrelations, which are substantial in both firing rates and fUSI signals. To obviate this problem, we estimated the optimal filter that relates the two through convolution (Boynton et al., 1996; Pisauro et al., 2013), using cross-validated ridge regression (Hoerl and Kennard, 1970). Convolving this filter with the firing rate yielded a prediction that closely matched the fUSI signal (Figure 1h). The convolved firing rate and the fUSI signal were highly correlated: in held-out data, the median correlation between the two was $\rho=0.49 \pm$ 0.13 (m.a.d., 34 recordings in 5 mice, Figure 1i).

The filter relating fUSI signals to firing rate had a half-width of $2.9 \mathrm{~s}$ and resembled the hemodynamic response function (HRF) characteristic of awake mouse cortex (Figure $\mathbf{1} \mathbf{j}-\mathbf{k}$ ). As expected, the estimated filter peaked with the same delay as the cross-correlations $(2.1 \pm 0.3 \mathrm{~s}$, median \pm m.a.d.), but it had a faster time-course. Its full-width at halfheight was $2.9 \pm 0.6 \mathrm{~s}$ (m.a.d., $34=$ experiments in 5 mice, Figure $1 \mathrm{j}$ ). Overall, the time course of the estimated filter closely resembled the fast hemodynamic response function measured optically in the cortex of awake mice (Pisauro et al., 2013; Figure 1k). The estimated filter, therefore, corresponds to the hemodynamic response function (HRF) of the cortex.

\section{Hemodynamic response function is similar across conditions and brain regions}

This simple linear relationship explained cortical fUSI signals not only during spontaneous activity but also during visually-driven activity (Figure 2a-d). To evoke visual responses, we presented a sequence of flashing checkerboards on the left, center, and right of the visual field (Figure 2a). In this sequence, the interval between stimuli was shorter than the time course of the HRF, so the fUSI signals did not have time to return to baseline between stimuli. Nonetheless, an event-related analysis of variations in activity relative to mean activity revealed the expected retinotopic maps in both primary visual cortex and superior colliculus, with left stimuli driving fUSI responses in the right hemisphere, center stimuli in both, and right stimuli in the left hemisphere (Brunner et al., 2020; Gesnik et al., 2017; Macé et al., 2018) (Figure 2b). Just as with spontaneous activity, the fUSI signal was well predicted by convolving the firing rate with the estimated HRF (Figure 2c,d). Across experiments the median correlation between convolved firing rate and fUSI signals, in held-out data, was $\rho=0.55$ \pm 0.22 (m.a.d. across 34 experiments in 5 mice).

The estimated HRF relating firing rate to fUSI signals was similar across mice, stimulus conditions, and brain regions (Figure $2 \mathrm{e}-\mathrm{g}$ ). The HRFs measured in visual cortex in different mice were similar, both during spontaneous activity (Figure 2e) and during visual stimulation (Figure 2f). Moreover, they resembled the HRFs measured in hippocampus (Figure 2g). To assess whether the same HRF applies across mice, stimulus conditions (visual stimulation vs. spontaneous activity), and brain regions (visual cortex vs. hippocampus), we compared the predictions of fUSI signals obtained while allowing different HRFs to predictions obtained while imposing a single average HRF. We used crossvalidation to avoid over-fitting. The single average HRF was just as good as the individual HRFs in predicting the responses. 


\section{a}

$$
\begin{aligned}
& \text { Visual } \\
& \text { stimuli }
\end{aligned}
$$

b

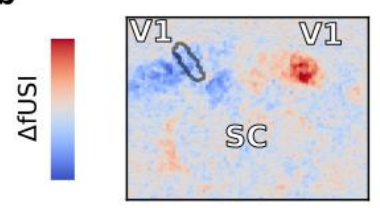

c

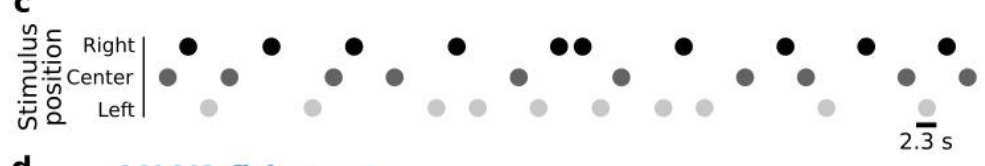

d

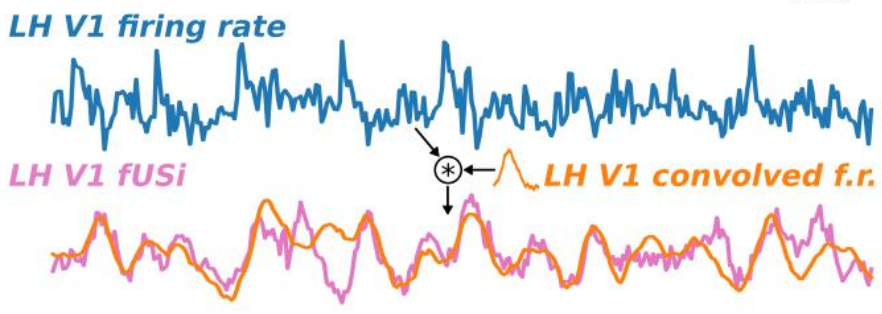

e Visual cortex
spontaneous activity

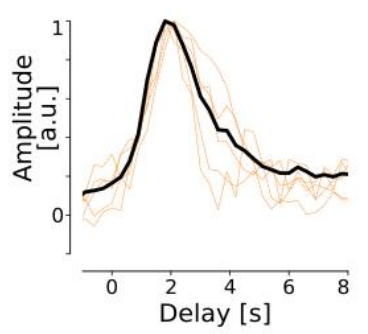

Visual cortex visual stimulation

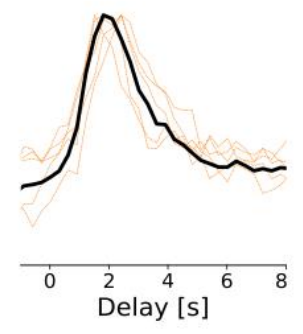

g Hippocampus

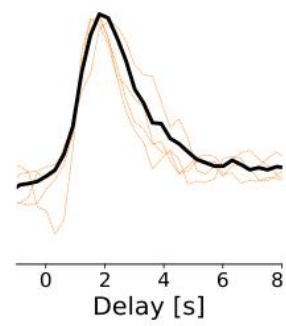

Figure 2. Hemodynamic response function is similar across conditions and brain regions. a. Flashing checkerboards were presented at three visual field locations: left, center and right. b. fUSI voxel responses to checkerboards. Black outline corresponds to voxels traversed by the Neuropixels probe sites located in visual cortex. Color scale indicates deviations from the mean activity during the stimulus. $\boldsymbol{c}-\boldsymbol{d}$. Mean response to sequence of 30 flashing checkerboards repeated 5 times. Visual cortex firing rate (blue) resembles fUSI time course (pink) after convolution with the hemodynamic response function estimated during visual stimulation (HRF, orange). e-f. The estimated HRFs for visual cortex under spontaneous activity and visual stimulation for individual mice (orange, $n=5$ ) were similar to the mean HRF computed across mice, areas and stimulus conditions (black). g. Individual HRFs for hippocampus estimated across spontaneous activity and visual stimulation (orange, $n=4$ ) were similar to the mean HRF (black, same as in $e-f)$.

\section{fUSI signals and firing rate are correlated across hemispheres}

Consistent with previous results, fUSI signals showed broad spatial correlations: activity in one location was highly correlated with activity at nearby locations and in the opposite hemisphere (Figure 3a,c,e,f,h,j). Similar to BOLD fMRI signals (Desjardins et al., 2001; Fox et al., 2006, 2007; Macey et al., 2004; Murphy et al., 2009), fUSI signals tend to have broad spatial correlations, especially across locations that are symmetrical across hemispheres (Ferrier et al., 2020; Osmanski et al., 2014; Rahal et al., 2020; Urban et al., 2015). Indeed, the fUSI traces measured in the left visual cortex during spontaneous activity correlated highly with fUSI signals measured in multiple other cortical and subcortical locations, including those in the opposite hemisphere (Figure 3a,c). Correlations between fUSI signals across hemispheres were as high as $\rho=0.75 \pm 0.08$ (median \pm m.a.d. across 68 recordings; Figure 3e, pink). Similar results were seen in the hippocampus, where the bilateral correlations were even higher, with values as high as $\rho=0.90 \pm 0.04$ for fUSI signals across hemispheres (across 58 recordings; Figure $3 f, h, j$ ).

Accordingly, the convolved firing rate correlated not only with fUSI signals at the same location but also at other locations, including those in the opposite hemisphere (Figure $\mathbf{3} b, c, d, g, i, j)$ ). The convolved firing rate measured in left visual cortex resembled not only the fUSI signals measured in the same location but also those measured in the opposite hemisphere (Figure $\mathbf{3 b}, \mathrm{d}$ ). Correlations with contralateral fUSI signals were $\rho=0.57 \pm 0.14$ (median \pm m.a.d.), barely lower than correlations with ipsilateral fUSI signals $(\rho=0.68 \pm 0.10)$. Likewise, the convolved firing rate measured in left hippocampus resembled fUSI signals measured in both left and right hippocampus (Figure $3 g, i, j$ ), with correlations above 0.7 in both cases (Figure $3 \mathrm{j}$ ). 




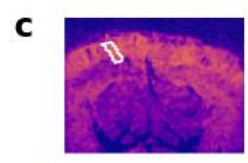

d



Correlation

h



e


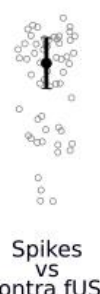

Figure 3. fUSI signals and firing rate are correlated across hemispheres. a. fUSI traces measured during spontaneous activity in an example recording, in a $\mathrm{ROI}$ in the left visual cortex (top) and in a symmetrical $\mathrm{ROI}$ in right visual cortex. $\boldsymbol{b}$. Convolved firing rate measured simultaneously in the left ROI. c. Correlation between the fUSI voxels in the left ROI (white contour) and all the individual fUSI voxels. $\boldsymbol{d}$. Correlation between the convolved firing rate measured in the left ROI (plus sign) and all the individual fUSI voxels. e. Left: Correlation between the fUSI traces measured from left and right visual cortex (as in a) in $n=68$ recordings during spontaneous activity and visual stimulation. Center, right: Correlations between the convolved firing rate in one hemisphere (as in b) and the fUSI traces (as in a) where firing rate and fUSI were measured simultaneously ( $n=68$ recordings). $f-j$. Same analyses for recordings where firing rate and fUSI were simultaneously measured in hippocampus ( $n=58$ recordings).

These results suggest that the strong spatial correlations seen in fUSI signals may be explained by underlying bilateral fluctuations in neural activity. Widefield imaging in mouse cortex has revealed that ongoing neural activity has broad spatial correlations and is strongly bilateral (Mohajerani et al., 2010; Musall et al., 2019; Shimaoka et al., 2019). The broadly correlated bilateral activity seen in fUSI signals, therefore, might be entirely neural in origin. However, there is another possible source of broad, bilateral noise: perhaps there are hemodynamic fluctuations that are broad and bilateral but not related to neuronal activity (Drew et al., 2020; Turner et al., 2020).

\section{Bilateral correlations in firing rate explain bilateral correlations in fUSI signals}

To investigate the high bilateral correlations observed in fUSI we performed simultaneous recordings with two Neuropixels probes and found high correlations between firing rates across hemispheres (Figure 4). In three of the mice we inserted two probes symmetrically relative to the midline, targeting bilateral locations in visual cortex and hippocampus (Figure 4a,d). During spontaneous activity, the convolved firing rate in left visual cortex closely resembled the one simultaneously recorded in right visual cortex (Figure $4 \mathrm{~b}$, orange). Across recordings, the two convolved firing rates had high bilateral covariance, with a correlation of $\rho=0.87 \pm 0.06$ (median \pm m.a.d., across 22 recordings; Figure 4c, orange). Similarly, the convolved firing rate measured in left and right hippocampus exhibited strong bilateral covariance, with a correlation of $\rho=0.93 \pm 0.03$ (median \pm m.a.d. across 14 recordings; Figure $4 d-f$ ). 
a

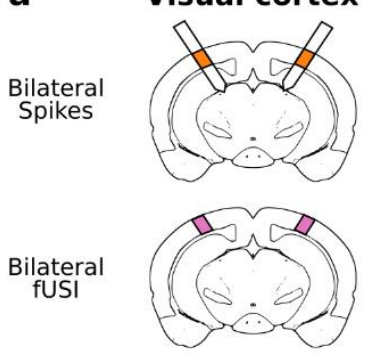

d Bilateral
Spikes

Bilateral
fUSI

\section{b}

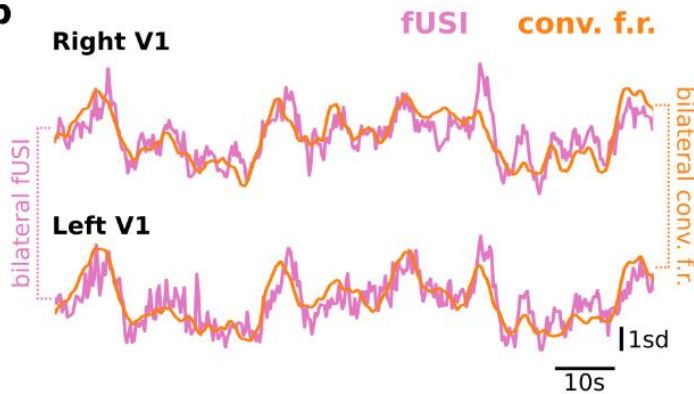

e

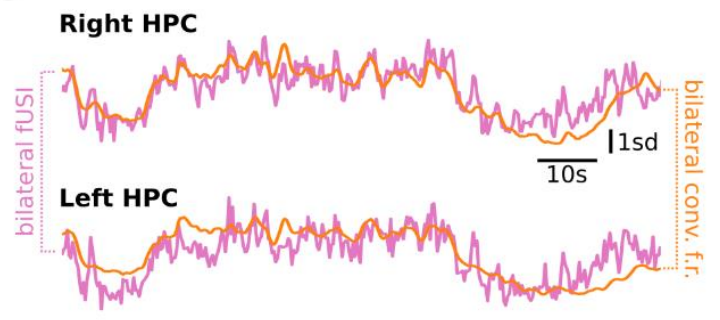

C



f

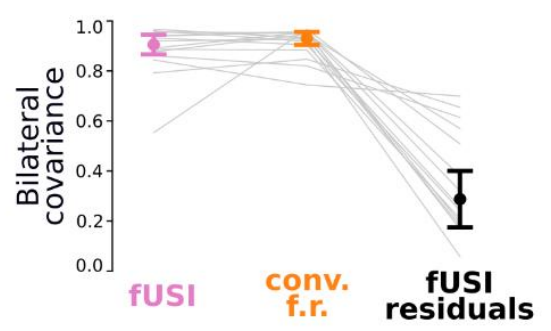

Figure 4. Bilateral correlations in firing rate explain bilateral correlations in fUSI signals. a. Neuropixels probes were inserted in each hemisphere targeting visual cortex and hippocampus. b. Example bilateral fUSI signals (pink) and convolved firing rate (orange) measured simultaneously from visual cortex during spontaneous activity. $c$. The convolved firing rate measured from left and right visual cortex exhibited strong covariance during spontaneous activity and visual stimulation (across 22 recordings in 3 mice; orange). The simultaneously recorded bilateral fUSI signals exhibited a similar level of covariance (pink). The contribution of non-neural factors to fUSI signals was estimated as the difference between the convolved firing rate and fUSI traces. After removing neural factors, the covariance between fUSI signals in the left and right hemispheres was substantially lower (black). $d$-f. Same analysis for hippocampus (across 14 recordings in 3 mice). The bilateral correlations in firing rate largely accounted for those measured in fUSI signals. The bilateral correlations measured in firing rate were as high as those measured in fUSI signals, both in visual cortex (paired $t$-test $P=0.28$, $n=22)$, and in hippocampus $(P=0.40, n=14)$. To test whether the bilateral correlations in firing rates fully explain the bilateral correlations observed in fUSI, we removed the fluctuations in $\mathrm{fUSI}$ signals that were predicted by the convolved firing rate measured at the same location and examined the residuals. The residuals had much smaller bilateral covariance than the original fUSI signals, both in visual cortex (paired t-test $P<10^{-10}, c$, black), and in hippocampus $\left(P<10^{-4}, f\right.$, black). These fUSI residuals strongly correlated with all voxels in the fUSI slice located inside the brain (not shown), suggesting that they reflect micromovements of the brain and global vascular effects possibly related to aliasing of respiratory and heartbeat signals.

\section{Discussion}

Much of brain activity is endogenous and unrelated to external events, so it must be measured in individual trials. Single-trial measurements of brain activity, however, are difficult with methods that have low signal/noise ratios, such as $\mathrm{fMRI}$ and EEG. These methods require recordings to be averaged across measurements by synchronizing to external or internal events (e.g. event-related analysis, or correlation with a seed voxel). At the other end of the spectrum are electrophysiology techniques that record neuronal spikes with such high signal/noise ratios that they routinely measure single-trial activity.

Our results indicate that functional ultrasound imaging is appropriate to measure brain activity in single trials. By performing simultaneous electrophysiology and functional ultrasound imaging, we were able to establish the relationship between neuronal firing and ultrasound signals on a trial-by-trial, moment-by-moment basis. The results indicate that functional ultrasound signals measured at frequencies below $0.3 \mathrm{~Hz}$ are largely neural in origin. Indeed, thanks to this high signal/noise ratio, fUSI signals can even be used to drive brain-machine interfaces (Norman et al., 2021).

Further, our double recording experiments reveal that fUSI signals match neural activity even when they spread over large portions of the brain, including the opposite hemisphere. fUSI signals commonly exhibit broad and laterally symmetric spatial correlations, and our results indicate that these are not due to measurement error or hemodynamic factors but rather are neural in origin. Large numbers of neurons are active at the 
same time, causing broad activations that are synchronous across space. These are typically associated with brain state and body movement (Drew et al., 2019; Musall et al., 2019; Stringer et al., 2019), and are often highly correlated across hemispheres (Drew, 2019; Fox et al., 2006, 2007; Mohajerani et al., 2010; Shimaoka et al., 2019). fUSI signals thus may appear noisy and broadly correlated, but their variability and correlations reflect true structured fluctuations in neuronal firing.

Finally, our results indicate that fUSI signals bear a simple relationship to the underlying neural activity captured by convolution with a standard hemodynamic response function. These results confirm and extend previous work that related blood signals to fUSI measurements performed separately and averaged across trials (Aydin et al., 2020; Boido et al., 2019). They indicate that the hemodynamic response function measured with fUSI is the same that had been measured optically (Pisauro et al., 2013), and is consistent across mice, stimulus conditions, and brain regions. However, we only tested two brain regions - visual cortex and hippocampus - and further investigations might reveal different hemodynamic responses elsewhere in the brain (Handwerker et al., 2004).

By releasing these data with simultaneous recordings and fUSI imaging, we hope to facilitate improvements to the processing pipeline that goes from raw Doppler images to fUSI signals. This pipeline includes multiple steps that aim to isolate signals related to neural activity from noise originating, e.g., from tissue movement (Baranger et al., 2018; Demené et al., 2015; Macé et al., 2011a, 2013). These steps include temporal high-pass filtering, principal component analysis, and subsequent removing of the components with the strongest amplitude. This pipeline is clearly already adequate, because it yields fUSI signals that are closely related to the underlying firing rates, but it may be amenable to further improvements. Moreover, it should be possible to design a deconvolution filter that estimates firing rate from fUSI signals, much as one can estimate firing rates from widefield calcium fluorescence (Peters et al.,
2021). For all this, it is essential to have the spikes of neurons as ground-truth data.

We conclude that fUSI signals bear a simple relationship to neuronal firing and accurately reflect this firing both in time and in space. We thus hope that our results will be useful to the increasing numbers of laboratories that are using functional ultrasound imaging to reveal the workings of the brain.

\section{Acknowledgments}

We thank Wang Qi for help with brain alignment and Célian Bimbard for helpful comments. This work was funded by a joint Wellcome Trust Investigator Award to $\mathrm{MC}$ and KHD (grant 205093/Z/16/Z). AU and GB were supported by grants from the Leducq Foundation (15CVD02), FWO (MEDI-RESCU2-AKUL/17/049, G091719N, and 1197818N), VIB Tech- Watch (fUSI-MICE), and the internal techfund from Neuro-Electronics Research Flanders (NERF). MC holds the GlaxoSmithKline / Fight for Sight Chair in Visual Neuroscience.

\section{Author Contributions}

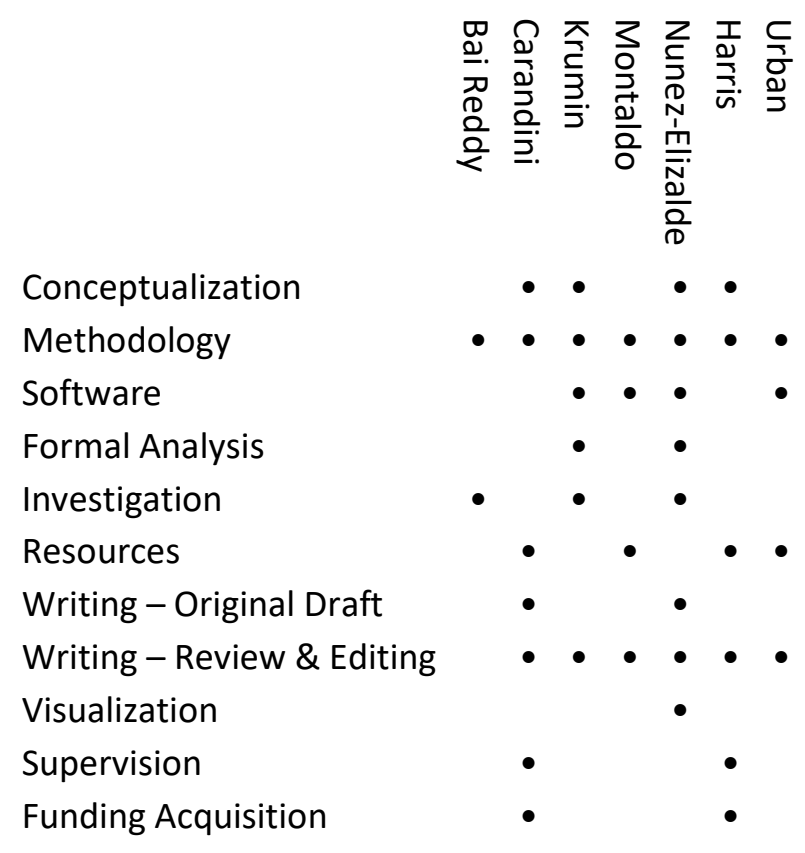

\section{Declaration of Interests}

A.U. is the founder and a shareholder of AUTC, a company commercializing neuroimaging solutions for preclinical and clinical research. 


\section{Methods}

All experimental procedures were conducted according to the UK Animals Scientific Procedures Act (1986). Experiments were performed at University College London, under a Project License released by the Home Office following appropriate ethics review.

\section{Initial surgery}

Experiments were conducted in 5 C57/BL6 mice of both sexes (4 male, 1 female), 9-12 weeks of age. Mice were first implanted with a cranial window under surgical anesthesia and in sterile conditions. The cranial window replaced a dorsal section of the skull ( $~ 8 \mathrm{~mm} \mathrm{ML}$ and $\sim 5 \mathrm{~mm}$ in AP) with $90 \mu \mathrm{m}$ thick ultrasound-permeable polymethylpentene (PMP) film (ME311070, Goodfellow Cambridge Ltd.). After the initial surgery, and between subsequent procedures, the PMP film was covered with KwikCast (World Precision Instruments, USA). This initial surgery was followed by 5-12 days of recovery, handling and habituation to the experimental rig.

\section{Recording sessions}

In each recording session, we fixed the cranial window to a post placed $10 \mathrm{~cm}$ from three computer screens (Adafruit, LP097QX1, 60Hz refresh rate) arranged at right angles to span 270 deg in azimuth and $~ 70$ deg in elevation. Fresnel lenses ( $f=220 \mathrm{~mm}$, BHPA220-2-5, Wuxi Bohai Optics) were mounted in front of the screens to reduce intensity differences across parts of the screens that are viewed from different angles. The lenses were covered with diffusing film (Frostbite, The Window Film Company) to reduce specular reflections.

We then inserted a Neuropixels probe (Jun et al., 2017) through a small hole in the PMP film $(0.5 \mathrm{~mm}$ radius). The probe described a parasagittal trajectory (posterolateral to anteromedial), at an angle of 28 deg relative to the midline (sagittal plane) and 40 deg relative to the horizontal (axial) plane. In some experiments we introduced a second Neuropixels probe in the opposite hemisphere, along the mirror-symmetric trajectory.

We then covered the PMP film with ultrasound gel and positioned an ultrasound transducer above it for fUSI acquisition (128-element linear array, 100 $\mu \mathrm{m}$ pitch, $8 \mathrm{~mm}$ focal length, $15 \mathrm{MHz}$ central frequency, model L22-Xtech, Vermon, France). The Doppler signals from the ultrasound transducer were acquired using a Vantage 128 ultrasound system (Verasonics, USA) controlled by a custom Matlab-based user interface (Alan Urban Consulting) and recording continuously at $500 \mathrm{~Hz}$. The fUSI acquisition was synchronized with the visual stimulus by recording the TTL pulses of the fUSI frames together with the flickering sync square on the visual stimulus monitor using Timeline feature of Rigbox (Bhagat et al., 2020). A similar method was used to align the Neuropixels recordings, by simultaneous recording of a randomly flickering external TTL pulses on an additional channel of a Neuropixels probe and on Timeline.

In each recording session, we moved the ultrasound transducer to cover 3-5 coronal slices. For each slice, we performed two recordings. In one recording we simply displayed a gray screen for $\sim 4$ minutes to measure spontaneous activity. In the other recording, we presented flashing checkerboards for $\sim 8$ minutes to measure stimulus evoked responses. The black and white checkerboards were presented in the left, center or right screens (one screen at a time). Checkerboards flashed at $2 \mathrm{~Hz}$ and each square had a size of $15 \mathrm{deg}$. The checkerboard sequence was interspersed with blank trials. The sequence consisted of 40 checkerboards, lasted $\sim 90$ seconds and was repeated 4-5 times.

At the end of the recording session we slowly extracted the Neuropixels probe from the brain while simultaneously recording fUSI images from one coronal slice. The movement induced by the probe extraction allowed us to localize the Neuropixels probe tip within the fUSI slice, giving us a 2D coronal projection of the probe's 3D trajectory.

Finally, we acquired a series of coronal fUSI images (a "Y-stack") from posterior to anterior, spaced 0.1 $\mathrm{mm}$ apart. These images were later used to construct a 3D fUSI volume of the brain to facilitate registration with the Allen Atlas and to identify the location of the Neuropixels probe in the fUSI slices. 


\section{Processing of ultrasound signals}

fUSI signals were computed using standard methods (Macé et al., 2011a). The $500 \mathrm{~Hz}$ complexvalued Doppler signals were divided into $400 \mathrm{~ms}$ chunks that overlapped by $50 \mathrm{~ms}$. Then, each chunk was high-pass filtered with a cut-off of $15 \mathrm{~Hz}$, and its principal components were computed in space and time. The first 15 principal components were then removed (Demené et al., 2015). A power Doppler image was then computed by squaring the complex-valued signals and averaging them in the central (non-overlapping) $300 \mathrm{~ms}$ window. The final temporal resolution of the fUSI signals was thus 3.33 Hz. The voxel time courses were then converted to percent signal change units relative to the mean of each voxel.

We computed the fUSI signal trace for a region of interest (ROI) by taking the mean of the individual time courses of voxels located within the ROI. The individual voxel time courses were normalized to percent signal change units before computing their mean.

fUSI images were manually aligned to vascular atlas with Allen CCF labels (Todorov et al., 2020). We first registered the 3D volume from each recording session to the vasculature atlas. To do this, the vasculature atlas was rotated, shifted and scaled to match the vasculature features salient in the fUSI $3 \mathrm{D}$ volume. These steps were performed using the FreeSurfer software package (Fischl, 2012). Once aligned, the transformation relating the vasculature atlas to the fUSI volume was saved and applied to the vasculature-matched Allen CCF labels. Finally, the Allen CCF labels were resampled to match the spatial resolution of the fUSI volume $(100 \times 100 \times 48$ $\mu \mathrm{m}^{3}$ ), yielding Allen CCF labels for each fUSI voxel.

\section{Spatial alignment}

To identify brain locations simultaneously traversed by the Neuropixels probe and the fUSI slices, we estimated the 3D trajectory of the Neuropixels probe within the fUSI Y-stack volume. Based on the geometry of the simultaneous recordings, we located the Neuropixels probe insertion site $\sim 0.2$ $\mathrm{mm}$ behind the posterior-most fUSI slice. We then computationally adjusted the Neuropixels probe 3D trajectory until its 2D coronal projection matched the 2D coronal projection measured with fUSI in vivo during Neuropixels probe extraction. This reconstructed 3D trajectory allowed us to map from Neuropixels probe sites to fUSI voxels in a slice, and vice versa.

While the Neuropixels probe intersects with the fUSI slice plane at one point in space, the fUSI slice has a thickness. The fUSI point spread function along the off-plane direction (i.e. the slice thickness) has a full-width at half maximum of $300 \mu \mathrm{m}$ (Brunner et al., 2020) and not larger than 500 um (Demené et al., 2016). For each recording, the fUSI voxels and Neuropixels probe sites located within $250 \mu \mathrm{m}$ on either side of the fUSI plane (along the $\mathrm{Y}$-axis) were used for the analyses.

For each recording, we identified the fUSI voxels that were intersected by the Neuropixels probe and used them to define a region of interest (ROI). The fUSI signal within the ROI was computed as the mean of the individual voxel time courses.

\section{Processing of electrophysiological signals}

The electrophysiology data was spike sorted and manually curated. We first spike sorted the data automatically using kilosort2 (Pachitariu et al., 2016). The resulting output was then manually curated with Phy (github.com/cortex-lab/phy) to identify clusters corresponding to single- and multiunit activity and to remove spurious and noisy clusters. After spike sorting, single- and multi-unit activity was summed across the electrode sites that traversed the fUSI imaging plane to obtain a single firing rate trace for a Region of Interest (ROI). This trace was binned at $\mathbf{3 0 0} \mathrm{ms}$ intervals to match the temporal resolution of fUSI signals.

To identify the Neuropixels probe sites located in visual cortex and in hippocampus, we used the cross-correlation of the multi-unit activity. We divided the Neuropixels probe sites into nonoverlapping $100 \mu \mathrm{m}$ segments and computed their cross-correlation. Sites at the top of the Neuropixels probe corresponded to visual cortex and were strongly correlated with each other. Sites immediately inferior to visual cortex corresponded to the hippocampus and were also strongly correlated with each other. 
To obtain ROIs in the fUSI images we identified the fUSI voxels traversed by the Neuropixels probe in visual cortex and hippocampus using the probe's 3D trajectory and a labeled volume of the standard C57 mouse brain, the Allen Common Coordinate Framework (Wang et al., 2020). For a ROI in given brain region (visual cortex or hippocampus) we included all voxels within the fUSI slice that were located within brain region (according to the Allen Common Coordinate Framework) and that were also located within the 2D projection of the Neuropixels probe trajectory.

\section{Cross-correlation and coherence}

The product-moment cross-correlation between firing rate and fUSI signal traces was computed at different delays by shifting the firing rate relative to the fUSI signals (from -5 to +30 seconds).

Coherence was computed using the multi-taper method (github.com/nipy/nitime). To do this, we used three minutes of firing rate and fUSI signal traces recorded simultaneously during periods of spontaneous activity. We computed the coherence between signals up to $1.667 \mathrm{~Hz}$, the Nyquist limit of our $300 \mathrm{~ms}$ sampling interval.

We computed the chance coherence between fUSI signals and firing rate at each frequency. To do this, we randomly and circularly shifted the firing rate and computed its coherence with the original fUSI signal trace. The null coherence was estimated by repeating this process 1,000 times and computing its mean at each frequency. The chance coherence across recordings was then computed as the median across recordings for each frequency.

To determine the highest frequency at which firing rate and fUSI signals are coherent, we compared the actual versus chance coherence values across sessions. To do this, we found the frequencies at which actual coherence was above chance coherence. We then identified the lowest frequency at which chance coherence was above the actual coherence $(0.32 \mathrm{~Hz})$. At this frequency and above, the actual coherence between firing rate and fUSI matches what can be expected by chance.

\section{Hemodynamic response function}

To estimate the hemodynamic response function relating firing rate to fUSI, we modelled fUSI responses for each recording were modeled was a linear combination of the firing rate in time using a finite impulse response filter. The model for each recording was estimated using estimated using cross-validated ridge regression (Hoerl and Kennard, 1970) using open-source software (Nunez-Elizalde et al., 2019). To avoid overfitting, the data was split into a training and a test set (75\%/25\%). Using the training set, the optimal regularization parameter was found independently in each recording using a 5-fold cross-validation procedure twice. The accuracy of the model was assessed by computing the correlation between predicted and actual fUSI signals in the held-out test set. Finally, the hemodynamic response function shape was estimated for each recording using $100 \%$ of the data.

\section{References}

Attwell, D., and ladecola, C. (2002). The neural basis of functional brain imaging signals. Trends Neurosci. 25, 621-625.

Aydin, A.K., Haselden, W.D., Goulam Houssen, Y., Pouzat, C., Rungta, R.L., Demené, C., Tanter, M., Drew, P.J., Charpak, S., and Boido, D. (2020). Transfer functions linking neural calcium to single voxel functional ultrasound signal. Nat. Commun. 11, $1-10$.

Baranger, J., Arnal, B., Perren, F., Baud, O., Tanter, M., and Demene, C. (2018). Adaptive Spatiotemporal SVD Clutter Filtering for Ultrafast Doppler Imaging Using Similarity of Spatial Singular Vectors. IEEE Trans. Med. Imaging 37, 15741586.

Bergel, A., Deffieux, T., Demené, C., Tanter, M., and Cohen, I. (2018). Local hippocampal fast gamma rhythms precede brainwide hyperemic patterns during spontaneous rodent REM sleep. Nat. Commun. 9.

Bhagat, J., Wells, M.J., Harris, K.D., Carandini, M., and Burgess, C.P. (2020). Rigbox: An open-source toolbox for probing neurons and behavior. eNeuro 7, 1-12.

Bimbard, C., Demene, C., Girard, C., Radtke-Schuller, S., Shamma, S., Tanter, M., and Boubenec, Y. (2018). Multi-scale mapping along the auditory hierarchy using high-resolution functional ultrasound in the awake ferret. eLife 7, 1-14.

Blaize, K., Arcizet, F., Gesnik, M., Ahnine, H., Ferrari, U., Deffieux, T., Pouget, P., Chavane, F., Fink, M., Sahel, J.A., et al. (2020). Functional ultrasound imaging of deep visual cortex in awake nonhuman primates. Proc. Natl. Acad. Sci. U. S. A. 117, 14453-14463. 
Boido, D., Rungta, R.L., Osmanski, B.F., Roche, M., Tsurugizawa, T., Le Bihan, D., Ciobanu, L., and Charpak, S. (2019). Mesoscopic and microscopic imaging of sensory responses in the same animal. Nat. Commun. 10, 1-13.

Boynton, G.M., Engel, S. a, Glover, G.H., and Heeger, D.J. (1996). Linear systems analysis of functional magnetic resonance imaging in human V1. J. Neurosci. 16, 4207-4221.

Brunner, C., Grillet, M., Sans-Dublanc, A., Farrow, K., Lambert, T., Macé, E., Montaldo, G., and Urban, A. (2020). A Platform for Brain-wide Volumetric Functional Ultrasound Imaging and Analysis of Circuit Dynamics in Awake Mice. Neuron 108, 861875.e7.

Buzsáki, G., Anastassiou, C. a, and Koch, C. (2012). The origin of extracellular fields and currents--EEG, ECoG, LFP and spikes. Nat. Rev. Neurosci. 13, 407-420.

Demené, C., Deffieux, T., Pernot, M., Osmanski, B., Biran, V., Gennisson, J., Sieu, L., Bergel, A., Franqui, S., Correas, J., et al. (2015). Spatiotemporal Clutter Filtering of Ultrafast Ultrasound Data Highly Increases Doppler and fUltrasound Sensitivity. IEEE Trans. Med. Imaging 34, 2271-2285.

Demené, C., Tiran, E., Sieu, L.A., Bergel, A., Gennisson, J.L., Pernot, M., Deffieux, T., Cohen, I., and Tanter, M. (2016). 4D microvascular imaging based on ultrafast Doppler tomography. Neuroimage 127, 472-483.

Desjardins, A.E., Kiehl, K.A., and Liddle, P.F. (2001). Removal of confounding effects of global signal in functional MRI analyses. Neuroimage 13, 751-758.

Devor, A., Ulbert, I., Dunn, A.K., Narayanan, S.N., Jones, S.R., Andermann, M.L., Boas, D.A., and Dale, A.M. (2005). Coupling of the cortical hemodynamic response to cortical and thalamic neuronal activity. Proc. Natl. Acad. Sci. U. S. A. 102, 3822-3827.

Dizeux, A., Gesnik, M., Ahnine, H., Blaize, K., Arcizet, F., Picaud, S., Sahel, J.A., Deffieux, T., Pouget, P., and Tanter, M. (2019). Functional ultrasound imaging of the brain reveals propagation of task-related brain activity in behaving primates. Nat. Commun. 10, 1-9.

Drew, P.J. (2019). Vascular and neural basis of the BOLD signal. Curr. Opin. Neurobiol. 58, 61-69.

Drew, P.J., Winder, A.T., and Zhang, Q. (2019). Twitches, Blinks, and Fidgets: Important Generators of Ongoing Neural Activity. Neuroscientist 25, 298-313.

Drew, P.J., Mateo, C., Turner, K.L., Yu, X., and Kleinfeld, D. (2020). Ultra-slow Oscillations in $\mathrm{fMRI}$ and Resting-State Connectivity: Neuronal and Vascular Contributions and Technical Confounds. Neuron 107, 782-804.

Ferrier, J., Tiran, E., Deffieux, T., Tanter, M., and Lenkei, Z. (2020). Functional imaging evidence for task-induced deactivation and disconnection of a major default mode network hub in the mouse brain. Proc. Natl. Acad. Sci. U. S. A. $117,15270-15280$.

Fischl, B. (2012). FreeSurfer. Neuroimage 62, 774-781.

Fox, M.D., Snyder, A.Z., Zacks, J.M., and Raichle, M.E. (2006). Coherent spontaneous activity accounts for trial-to-trial variability in human evoked brain responses. Nat. Neurosci. 9, 23-25.

Fox, M.D., Snyder, A.Z., Vincent, J.L., and Raichle, M.E. (2007). Intrinsic Fluctuations within Cortical Systems Account for Intertrial Variability in Human Behavior. Neuron 56, 171-184.

Gesnik, M., Blaize, K., Deffieux, T., Gennisson, J.L., Sahel, J.A., Fink, M., Picaud, S., and Tanter, M. (2017). 3D functional ultrasound imaging of the cerebral visual system in rodents. Neuroimage 149, 267-274.

Hamel, E. (2006). Perivascular nerves and the regulation of cerebrovascular tone. J. Appl. Physiol. 100, 1059-1064.

Handwerker, D. a., Ollinger, J.M., and D’Esposito, M. (2004). Variation of BOLD hemodynamic responses across subjects and brain regions and their effects on statistical analyses. Neuroimage 21, 1639-1651.

Heeger, D.J., and Ress, D. (2002). What does fMRI tell us about neuronal activity? Nat. Rev. Neurosci. 3, 142-151.

Hillman, E.M.C. (2014). Coupling mechanism and significance of the BOLD signal: A status report. Annu. Rev. Neurosci. 37, 161181.

Hoerl, A.E., and Kennard, R.W. (1970). Ridge regression: Biased estimation for nonorthogonal problems. Technometrics 12 , 55-67.

ladecola, C., and Nedergaard, M. (2007). Glial regulation of the cerebral microvasculature. Nat. Neurosci. 10, 1369-1376.

Jun, J.J., Steinmetz, N.A., Siegle, J.H., Denman, D.J., Bauza, M., Barbarits, B., Lee, A.K., Anastassiou, C.A., Andrei, A., Aydın, Ç., et al. (2017). Fully integrated silicon probes for high-density recording of neural activity. Nature 551, 232.

Katzner, S., Nauhaus, I., Benucci, A., Bonin, V., Ringach, D.L., and Carandini, M. (2009). Local Origin of Field Potentials in Visual Cortex. Neuron 61, 35-41.

Koekkoek, S.K.E., Soloukey Tbalvandany, S., Generowicz, B.S., van Hoogstraten, W.S., de Oude, N.L., Boele, H.J., Strydis, C., Leus, G., Bosch, J.G., van der Steen, A.F.W., et al. (2018). High Frequency Functional Ultrasound in Mice. In 2018 IEEE International Ultrasonics Symposium (IUS), pp. 1-4.

Logothetis, N.K., Pauls, J., Augath, M., Trinath, T., and Oeltermann, A. (2001). Neurophysiological investigation of the basis of the fMRI signal. Nature 412, 150-157.

Macé, É., Montaldo, G., Cohen, I., Baulac, M., Fink, M., and Tanter, M. (2011a). Functional ultrasound imaging of the brain. Nat. Methods 8, 662-664.

Macé, É., Montaldo, G., Cohen, I., Baulac, M., Fink, M., and Tanter, M. (2011b). Functional ultrasound imaging of the brain. Nat. Methods 8, 662-664.

Macé, É., Montaldo, G., Osmanski, B.F., Cohen, I., Fink, M., and Tanter, M. (2013). Functional ultrasound imaging of the brain: Theory and basic principles. IEEE Trans. Ultrason. Ferroelectr. Freq. Control 60, 492-506.

Macé, É., Montaldo, G., Trenholm, S., Cowan, C., Brignall, A., 
Urban, A., and Roska, B. (2018). Whole-Brain Functional Ultrasound Imaging Reveals Brain Modules for Visuomotor Integration. Neuron 100, 1241-1251.e7.

Macey, P.M., Macey, K.E., Kumar, R., and Harper, R.M. (2004). A method for removal of global effects from $\mathrm{fMRI}$ time series. Neuroimage 22, 360-366.

Martindale, J., Mayhew, J., Berwick, J., Jones, M., Martin, C., Johnston, D., Redgrave, P., and Zheng, Y. (2003). The hemodynamic impulse response to a single neural event. J. Cereb. Blood Flow Metab. 23, 546-555.

Mohajerani, M.H., McVea, D.A., Fingas, M., and Murphy, T.H. (2010). Mirrored bilateral slow-wave cortical activity within local circuits revealed by fast bihemispheric voltage-sensitive dye imaging in anesthetized and awake mice. J. Neurosci. 30, 3745-3751.

Murphy, K., Birn, R.M., Handwerker, D.A., Jones, T.B., and Bandettini, P.A. (2009). The impact of global signal regression on resting state correlations: Are anti-correlated networks introduced? Neuroimage 44, 893-905.

Musall, S., Kaufman, M.T., Juavinett, A.L., Gluf, S., and Churchland, A.K. (2019). Single-trial neural dynamics are dominated by richly varied movements. Nat. Neurosci. 22, 1677-1686.

Nair, D.G. (2005). About being BOLD. Brain Res. Brain Res. Rev. $50,229-243$.

Norman, S., Maresca, D., Christopoulos, V., Griggs, W., Demene, C., Tanter, M., Shapiro, M., and Andersen, R. (2021). Single Trial Decoding of Movement Intentions Using Functional Ultrasound Neuroimaging. Neuron 1-13.

Nunez-Elizalde, A.O., Huth, A.G., and Gallant, J.L. (2019). Voxelwise encoding models with non-spherical multivariate normal priors. Neuroimage 197, 482-492.

Osmanski, B.F., Pezet, S., Ricobaraza, A., Lenkei, Z., and Tanter, $M$. (2014). Functional ultrasound imaging of intrinsic connectivity in the living rat brain with high spatiotemporal resolution. Nat. Commun. 5.

Pachitariu, M., Steinmetz, N., Kadir, S., Carandini, M., and Harris, K. (2016). Fast and accurate spike sorting of highchannel count probes with KiloSort. Adv. Neural Inf. Process. Syst. 4455-4463.

Peters, A.J., Fabre, J.M.J., Steinmetz, N.A., Harris, K.D., and Carandini, M. (2021). Striatal activity topographically reflects cortical activity. Nature 591.

Pisauro, M.A., Dhruv, N.T., Carandini, M., and Benucci, A. (2013). Fast Hemodynamic Responses in the Visual Cortex of the Awake Mouse. J. Neurosci. 33, 18343-18351.

Provansal, M., Labernède, G., Joffrois, C., Rizkallah, A., Goulet, R., Valet, M., Deschamps, W., Ferrari, U., Chaffiol, A., Dalkara, D., et al. (2021). Functional ultrasound imaging of the spreading activity following optogenetic stimulation of the rat visual cortex. BioRxiv 2021.02.05.429985.

Rabut, C., Yoo, S., Hurt, R.C., Jin, Z., Li, H., Guo, H., Ling, B., and Shapiro, M.G. (2020). Ultrasound Technologies for Imaging and
Modulating Neural Activity. Neuron 108, 93-110.

Rahal, L., Thibaut, M., Rivals, I., Claron, J., Lenkei, Z., Sitt, J.D., Tanter, M., and Pezet, S. (2020). Ultrafast ultrasound imaging pattern analysis reveals distinctive dynamic brain states and potent sub-network alterations in arthritic animals. Sci. Rep. 10, $1-17$.

Rubin, J.M., Bude, R.O., Carson, P.L., Bree, R.L., and Adler, R.S. (1994). Power Doppler US: A potentially useful alternative to mean frequency- based color Doppler US. Radiology 190, 853856.

Rubin, J.M., Adler, R.S., Fowlkes, J.B., Spratt, S., Pallister, J.E., Chen, J.F., and Carson, P.L. (1995). Fractional moving blood volume: Estimation with power Doppler US. Radiology 197, 183-190.

Sans-Dublanc, A., Chrzanowska, A., Reinhard, K., Lemmon, D., Montaldo, G., Urban, A., and Farrow, K. (2020). Brain-wide mapping of neural activity mediating collicular-dependent behaviors. BioRxiv.

Schölvinck, M.L., Saleem, A.B., Benucci, A., Harris, K.D., and Carandini, M. (2015). Cortical state determines global variability and correlations in visual cortex. J. Neurosci. 35, 170178.

Shimaoka, D., Steinmetz, N.A., Harris, K.D., and Carandini, M. (2019). The impact of bilateral ongoing activity on evoked responses in mouse cortex. eLife 8, 1-19.

Sieu, L.A., Bergel, A., Tiran, E., Deffieux, T., Pernot, M., Gennisson, J.L., Tanter, M., and Cohen, I. (2015). EEG and functional ultrasound imaging in mobile rats. Nat. Methods 12, 831-834.

Stringer, C., Pachitariu, M., Steinmetz, N., Reddy, C.B., Carandini, M., and Harris, K.D. (2019). Spontaneous behaviors drive multidimensional, brainwide activity. Science (80-. ). 364.

Todorov, M.I., Paetzold, J.C., Schoppe, O., Tetteh, G., Shit, S., Efremov, V., Todorov-Völgyi, K., Düring, M., Dichgans, M., Piraud, M., et al. (2020). Machine learning analysis of whole mouse brain vasculature. Nat. Methods 17, 442-449.

Turner, K.L., Gheres, K.W., Proctor, E.A., and Drew, P.J. (2020). Neurovascular coupling and bilateral connectivity during nrem and rem sleep. eLife $9,1$.

Urban, A., Dussaux, C., Martel, G., Brunner, C., Mace, E., and Montaldo, G. (2015). Real-time imaging of brain activity in freely moving rats using functional ultrasound. Nat. Methods $12,873-878$.

Wang, Q., Ding, S.L., Li, Y., Royall, J., Feng, D., Lesnar, P., Graddis, N., Naeemi, M., Facer, B., Ho, A., et al. (2020). The Allen Mouse Brain Common Coordinate Framework: A 3D Reference Atlas. Cell 181, 936-953.e20.

Winder, A.T., Echagarruga, C., Zhang, Q., and Drew, P.J. (2017). Weak correlations between hemodynamic signals and ongoing neural activity during the resting state. Nat. Neurosci. 20, 17611769. 
bioRxiv preprint doi: https://doi.org/10.1101/2021.03.31.437915; this version posted March 31, 2021. The copyright holder for this preprint (which was not certified by peer review) is the author/funder, who has granted bioRxiv a license to display the preprint in perpetuity. It is made available under aCC-BY 4.0 International license. 